\title{
Growth and Microstructural Properties of Nitride-Based Nanostructures
}

\author{
Y.H. Kim, ${ }^{*}$ H. Ryu*, Y.H. Shin, ${ }^{* *}$ and M.D. Kim** \\ * Division of Industrial Metrology, Korea Research Institute of Standards and Science, 209
}

Gajeong-Ro, Yuseong-Gu, Daejeon 305-340, Republic of Korea

** Department of Physics, Chungnam National University, 220 Gung-Dong, Yuseong-Gu, Daejeon, 305-764, Republic of Korea

Group III-nitrides have been recognized to be a great variety of applications such as UV photodetectors, laser diodes, and high-speed field-effect transistors due to their direct band gap, emissive property, and extremely large heterojunction offsets. In addition, nanometer-sized low dimensional III-nitrides are expected to improve the device characteristics and to be used as building block for nanodevices because they can have quantum confinement effects and be free from defects. Exactly, various III-nitride nanostructures such as nanowire, nanorod, nanobelt, and nanosheet have been successfully synthesized by various deposition techniques such as simple gas reaction, arc discharge, pyrolysis, laser ablation, hydride vapor phase epitaxy (HVPE), and chemical vapor deposition (CVD).

We have reported the growths of $\mathrm{GaN}$ nanowires and indium-related novel architectures by molecular beam epitaxy (MBE) [1, 2]. Because MBE system is possible to precise control of the beam fluxes and growth conditions, it will be a powerful facility for nanostructures and nanodevices. Recently, the InN/GaN heterostructured nanowires (HNs) were successfully grown on $\mathrm{Si}(111)$ substrate by molecular beam epitaxy method without catalyst aid. In this presentation, we report the growth behavior and microstructural properties of the InN/GaN HNs. Their detailed structural characteristics were studied by X-ray diffraction analysis and electron microscopy study. Based on those structural properties, the growth mechanism of HNs is demonstrated.

Figure 1 shows the cross-sectional and plan-view SEM images for the InN/GaN HNs grown on $\mathrm{Si}$ (111) substrate. The nanowires have identical morphology, and all the nanowires are freestanding and well aligned. The planar image in Fig. 1(c) indicates that the HNs have polygonal prismatic shapes, mostly, hexagonal prism.In Figure 2 (a), which is the cross-sectional HRTEM micrograph of $\mathrm{GaN} / \mathrm{Si}(111)$ interface, a thin amorphous $\mathrm{Si}_{\mathrm{x}} \mathrm{N}_{\mathrm{y}}$ layer of below $2 \mathrm{~nm}$ in thickness developed at the interface. Although the $\mathrm{Si}_{\mathrm{x}} \mathrm{N}_{\mathrm{y}}$ layer exists at the interface, the alignment of the nanowires with its $c$ axis perpendicular to the substrate is observed. In the analysis of the SAED pattern, the epitaxial relationship between the $\mathrm{GaN}$ nanowire and the $\mathrm{Si}(111)$ substrate is maintained to $\mathrm{GaN}(0001) / / \mathrm{Si}(111)$ and $\mathrm{GaN}[2110] / / \mathrm{Si}[110]$ (Fig. 2b). Fig. 2c show the bright field image of the 3dimensional (3-D) GaN nanoisland. The 3-D nanoislands have a triangular shape.

The crystallographic properties of the InN nanowires were determined by those of the GaN nanowires. The GaN 3-D nanoislands were observed at the interface. From these observations, it is deduced that the competition between the nanowires and the 3-D nanoislands was caused at the initial growth stage.

References

[1] Y.H. Kim, J.Y. Lee, S.H. Lee, J.E. Oh, and H.S. Lee, Appl. Phys. A 80 (2005) 1635.

[2] Y.H. Kim, J.Y. Lee, S.H. Lee, J.E. Oh, H.S. Lee, Y. Huh, Chem. Phys. Lett. 412 (2005) 454. 
[3] This work is supported by Korea Research Council of Fundamental Science and Technology (KRCF) through the project of "Development of Characterization Techniques for Nanomaterials Safety".

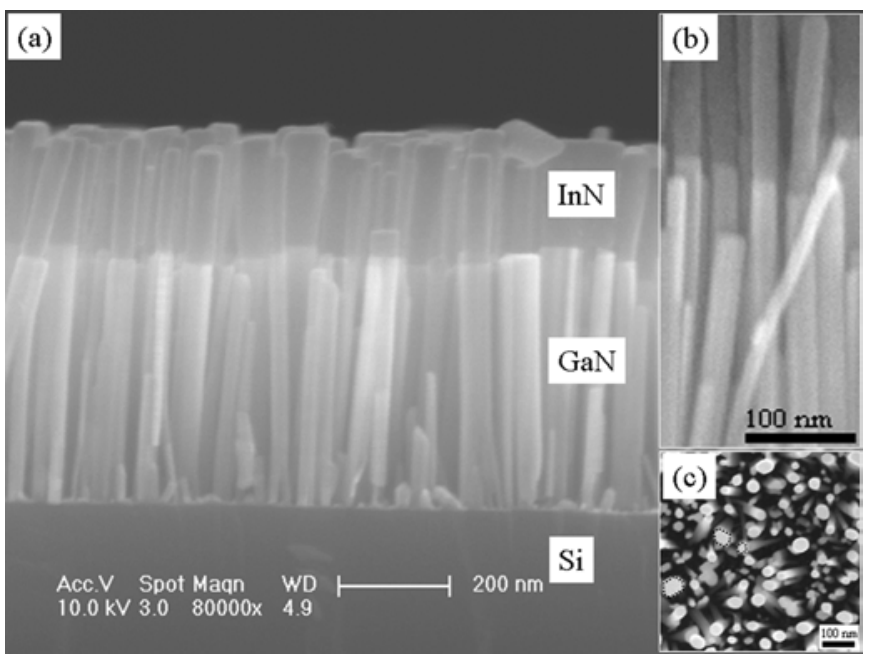

FIG. 1. (a) Cross-sectional FESEM image of the InN/GaN heterostructured nanowires (HNs). (b) Magnified image. (c) Plane-view FESEM image of the HNs.

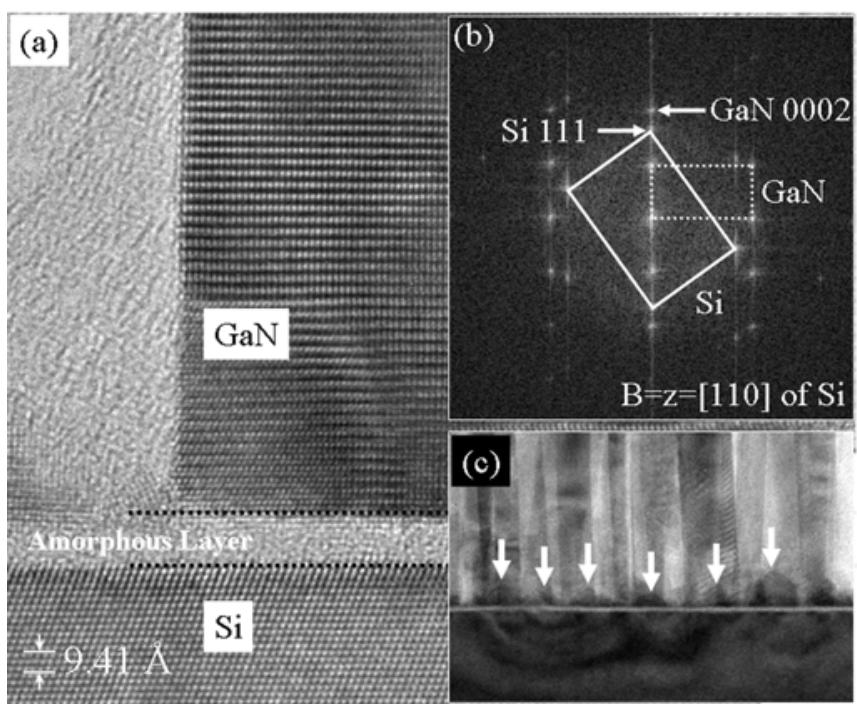

FIG. 2. . (a) Cross-sectional HRTEM micrograph of the GaN/Si(111) interface revealing the thin $\mathrm{Si}_{\mathrm{x}} \mathrm{N}_{\mathrm{y}}$ layer developed at the interface between the GaN and the bare Si substrate. (b) SAED pattern obtained by fast Fourier transform (FFT) of the lattice image in (a). (c) Cross-sectional BF image of the GaN/Si interface. The arrows indicate nanometer-scale 3D islands. 